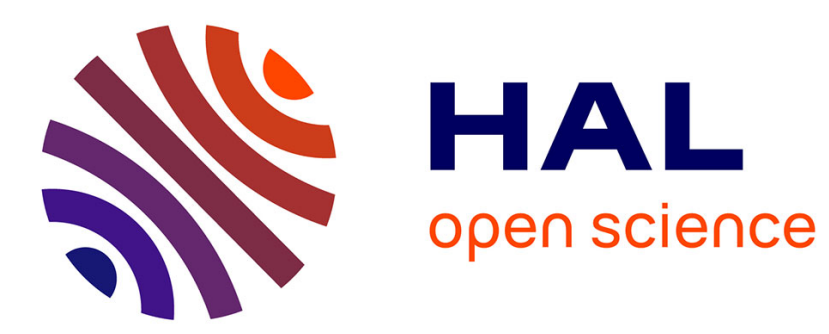

\title{
Efficient ACC with Stop\&Go Maneuvers for Hybrid Vehicle with Online Sub-Optimal Energy Management
}

Rustem Abdrakhmanov, Lounis Adouane

\section{To cite this version:}

Rustem Abdrakhmanov, Lounis Adouane. Efficient ACC with Stop\&Go Maneuvers for Hybrid Vehicle with Online Sub-Optimal Energy Management. 11th International Workshop on Robot Motion and Control, Jul 2017, Wasowo, Poland. hal-01659683

\section{HAL Id: hal-01659683 \\ https://hal.science/hal-01659683}

Submitted on 27 Feb 2018

HAL is a multi-disciplinary open access archive for the deposit and dissemination of scientific research documents, whether they are published or not. The documents may come from teaching and research institutions in France or abroad, or from public or private research centers.
L'archive ouverte pluridisciplinaire HAL, est destinée au dépôt et à la diffusion de documents scientifiques de niveau recherche, publiés ou non, émanant des établissements d'enseignement et de recherche français ou étrangers, des laboratoires publics ou privés. 


\title{
Efficient ACC with Stop\&Go Maneuvers for Hybrid Vehicle with Online Sub-Optimal Energy Management*
}

\author{
Rustem Abdrakhmanov and Lounis Adouane
}

\begin{abstract}
This paper presents an Adaptive Cruise Control with Stop\&Go (ACCwSG) maneuvers aiming to improve the fuel economy of a Hybrid Electric Vehicle (HEV). Within the scope of the given subject, an optimal offline optimization, based on Dynamic Programming (DP), is proposed in this work, permitting to have simultaneous speed profile optimization and optimal power split strategy of a series-parallel hybrid bus. Afterwards, the basis of the offline optimal strategy has been adapted in order to deal online with the current road profile and driver velocity demand. The proposed online and suboptimal strategy uses mainly a Multi-Dimensional Database (MDD, which gives as output the speed profile and powersplit set-points) and an appropriate interpolation technique in order to cope with the current bus situations. The MDD is obtained based on offline DP, while using specific protocol which cover a large possible space state of the vehicle situations and their corresponding optimal set-points. The developed strategy finds its practical application in ACCwSG for a heavy urban bus, which is subject to frequent highly energy consuming starts and stops phases. The present work is conducted on a dedicated high-fidelity dynamical model of the hybrid bus that was developed on MATLAB/TruckMaker software. Several simulations were performed to prove the efficiency of the proposed navigation strategy.
\end{abstract}

\section{INTRODUCTION}

The problem of reducing the environment pollution in order to save the planet has become one of the most important challenges in the world. Besides, the worldwide crisis of the fossil fuel resources, which diminish at high rate, aggravates it. These two global aspects made the big industrial companies and the state governments invest increasingly into the alternative energy sources. The hybrid electric vehicles (HEV) promise a relevant solution with regard to the objectives of reducing the fuel consumption, as well as the decrease of the exhaust gazes emission [1].

In urban environment, due to traffic conditions, traffic lights, a bus encounters frequent Stop\&Go situations. This results in augmented fuel consumption during the starts. The advantage of the HEV is that during the deceleration phases a part of the energy can be recuperated to recharge the electric battery. In this sense, the ACCwSG strategy brings the undeniable benefit. The acceleration and deceleration profiles and energy management strategy can help to reduce the fuel consumption.

Some authors have started to explore the introduction of additional objectives to ACC, e.g., fuel economy and driver

*This project is supported by the ADEME (Agence De l'Environnement et de la Matrise de l'Energie) for the National French program "Investissement d'Avenir", through BUSINOVA Evolution project.

Institut Pascal / IMobS3, UCA/SIGMA UMR CNRS 6602, ClermontFerrand, France. e-mail: Firstname. Lastname@uca. fr desired response. [2] proposed a DP based offline control method. It reduces the fuel consumption while allowing reliable tracking error (preceding car speed tracking). The improvement of fuel consumption usually decreases the acceleration performance and lowers the tracking capability. If an ACC system pursues good tracking capability only, it leads to unnecessary acceleration and emergency braking, which deteriorates the fuel economy of vehicle to some extent. A velocity control system in order to save the fuel consumption by involving traffic signal information was proposed in [3]. Model predictive control scheme was used in order to control the velocity predicting states of the vehicle and traffic signal switching. The algorithm judges whether a vehicle should accelerate or not when the vehicle cannot pass the traffic lights during the green phase. In the algorithm, the fuel economy was predicted using traffic signal information. A vehicle speed and vehicle-to-vehicle distance control algorithm for vehicle stop\&go cruise control has been proposed in [4]. [5] formulated the optimization problem to find the optimal relative distance profile during a complete stop, and the optimal velocity profile during a starting motion. Linear quadratic optimal control theory has been used to develop a vehicle speed and distance control algorithm. A desired acceleration for the vehicle has been designed on the basis of the vehicle speed and distance control algorithm. It suggested that once leader car resumes the motion after a full stop, the host car is not obliged to follow the leader car, instead it can follow an optimal profile.

Concerning the energy optimization in the HEV and pure electric vehicles (EV), the researches mainly deal with two kind of problems: 1) energy power management for a given velocity profile [6][7][8][9]; 2) velocity profile optimization for EV or conventional vehicles [10][11][12]. The application of the optimal control theory to power management on HEV has been the most popular approach, which includes linear programming, optimal control and especially DP [6] [7] [13] [14][15]. These techniques have been widely studied and applied to a broad range of vehicles.

Unlike the previous publications, the present paper proposes ACCwSG, which improves the fuel economy and provides passengers' comfort. It is carried out, first, by developing an optimal offline optimization based on DP permitting to have simultaneous speed profile optimization and optimal power split strategy of the studied series-parallel hybrid bus, aiming to reduce the fuel and electrical energy consumption. For an urban bus the route is normally known in advance, so the optimization is performed for given road profile. The Predictive battery State of the Charge (P-SOC) 
block is introduced in order to guarantee that at the end of operational cycle (generally, a full day cycle) the electric battery charge is not below its minimum permitted level. Another important aspect of the public transportation is the passengers comfort. For that purpose the maximal allowed acceleration and deceleration are taken into account. As the hybrid bus' electric motor and engine have different dynamic characteristics (the power supplied, the response time, etc.), the dynamic constraints linked to the dynamics of the motors are taken into account. DP is highly time consuming, that is why a specific protocol was proposed in order to perform an online sub-optimal strategy. An online sub-optimal speed profile and related power split generation using different multi-dimensional interpolation methods is developed to deal online with the current road profile and driver velocity demand. This is carried out using the Optimal Profile Database based on DP (OPD-DP) applied to the ACCwSG control strategy.

The rest of the paper is organized as follows. In section II, the studied bus powertrain and its dynamical model are presented. Section III presents the control scheme of the ACCwSG. Section IV presents the proposed sub-optimal ACCwSG based on DP. In section V several simulation results are presented showing the efficiency of the proposed velocity profile optimization and energy management strategies for ACCwSG. Finally, conclusions and some prospects are given in the last section.

\section{MODELING OF THE HYBRID BUS}

The aim of this section is to illustrate the architecture and the mathematical model of the studied system, i.e., BUSINOVA hybrid bus, developed by SAFRA company (cf. figure 1) $)^{1}$. This bus is composed of an electric motor, a hydraulic motor, an internal combustion engine and battery as the propulsion powertrain system of the vehicle.

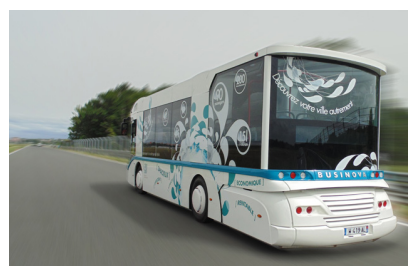

Fig. 1. BUSINOVA hybrid bus

\section{A. Hybrid bus powertrain architecture}

The model of the studied hybrid bus is based on a series-parallel power-split hybrid architecture [16]. A simple block diagram of the power flows in the bus is shown in figure 2. The electric (EM) and hydraulic (HM) motors are both directly connected to the transmission and can ensure simultaneously or independently the traction of the bus. On the other hand, the internal combustion engine (ICE) is coupled to a hydraulic pump (HP) for driving the HM, and therefore allowing the ICE load shifting.

\footnotetext{
${ }^{1}$ http://www.businova.com
}

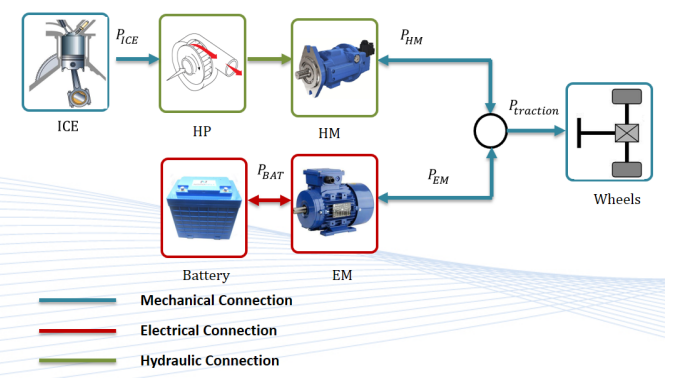

Fig. 2. Block diagram of the powertrain power flows. (ICE: internal combustion engine, HP: hydraulic pump, HM: Hydraulic motor, EM: electric motor)

The rotational speeds of the HM and the EM are imposed by the wheels speed in proportion to the reduction ratios of HM and EM respectively. Moreover, the rotational speed $\omega_{H M}$ and the torque $T_{H M}$ are expressed as follows:

$$
\left\{\begin{array}{l}
\omega_{H M}\left(T_{I C E}, D_{H M}\right)=\frac{D_{H P} \cdot \eta_{v_{H M}} \cdot \omega_{I C E}}{D_{H M} \cdot \eta_{v_{H} P}} \\
T_{H M}\left(T_{I C E}, D_{H M}\right)=\frac{D_{H M} \cdot \eta_{m_{H M}} \cdot T_{I C E}}{D_{H P} \cdot \eta_{m_{H P}}}
\end{array}\right.
$$

where $\omega_{I C E}, T_{I C E}$ are respectively rotational speed and torque of the ICE, and $D_{H M}, D_{H P}, \eta_{m_{H M}}, \eta_{m_{H P}}, \eta_{v_{H M}}$, $\eta_{v_{H P}}$ are respectively displacement, mechanical efficiency and volumetric efficiency of the HM and the HP.

The BUSINOVA can operate according to the modes described below:

1) the propulsion is fully supplied by the electric motor (mode I),

2) the bus is actuated by the hydraulic motor via the ICE (mode II),

3) the mode III implies the hybrid operation of the EM and the HM via ICE,

4) the regenerative braking (mode IV) - the part of the kinetic energy during braking phase is recuperated to charge the electric battery.

\section{B. Dynamical model}

This part is dedicated to the dynamical equations describing the bus. The purpose of the dynamical model is to have a realistic global behavior of the bus in order to validate the proposed energy management techniques. To describe it in a generic manner, assume that the bus is moving up the slope of $\theta$ degree (cf. figure 4). The origin of the coordinates is situated in the Center of Mass (CoM). It is supposed that $\mathrm{CoM}$ of the bus is in its geometric center. The dynamical equation of the bus is given as follows:

$$
\vec{F}_{t r}+\vec{F}_{r r}+\vec{F}_{a d}+\vec{F}_{g}+\vec{F}_{\text {brake }}=\left(M+M_{e q}\right) \vec{a}
$$

where $\vec{F}_{t r}$ traction force, $\vec{F}_{r r}$ rolling resistance, $\vec{F}_{a d}$ aerodynamic force, $\vec{F}_{g}$ gravity force, $\vec{F}_{\text {brake }}$ mechanical brake force, $M$ bus weight, $M_{e q}$ equivalent mass of rotating parts, $\vec{a}$ bus acceleration.

To produce the bus acceleration, it is necessary to take into account the moments of inertia of the rotating components 
(e.g., rotor of the EM, crankshaft of the ICE, driving axle, etc.). It is done by introducing the equivalent mass $M_{e q}$ of the rotating components:

$$
M_{e q}=\frac{i_{g} \eta_{p t} J_{r o t}}{r^{2}}
$$

where $i_{g}$ gear ratio, $\eta_{p t}$ powertrain efficiency, $J_{\text {rot }}$ total inertia of the rotating components in the transmission, and $r$ the wheel radius [17].

The traction force $F_{t r}$ is linked to the torque produced by the powertrain $T_{p t}$ via gear ratio $i_{g}$, powertrain efficiency $\eta_{p t}$. Expanding the dynamical equation (2), the following relation is obtained:

$$
a=\frac{d v}{d t}=\frac{1}{M+M_{e q}} H
$$

with

$$
\begin{array}{r}
H=\frac{i_{g} \eta_{p t} T_{p t}}{r}-\mu_{r r} F_{N} \operatorname{sign}(v)-\frac{1}{2} \rho A C_{d}\left(v+v_{w i n d}\right)^{2} \\
-M g \sin (\theta)-\frac{T_{\text {brake }}}{r}
\end{array}
$$

where:

- $T_{p t}$ : output powertrain torque from the gearbox,

- $\mu_{r r}$ : rolling resistance coefficient, $F_{N}=M g \cos (\theta)$ normal force, $g$ gravity acceleration, $\theta$ slope angle, $v$ bus speed,

- $\rho$ : the air density, $A$ the frontal area of the bus, $C_{d}$ drag coefficient, $v_{\text {wind }}$ wind speed,

- $T_{\text {brake }}$ : the brake torque provided by the bus mechanical brake system.

\section{Adaptive Cruise Control With Stop\&Go}

Adaptive Cruise Control with Stop\&Go (ACCwSG) is a mix of vehicle capability of maintaining a user-preset speed (Cruise Control), capability of keeping a save distance from a vehicle ahead (ACC) and capability to perform maneuvers at low speeds (Stop\&Go) (cf. figure 3).

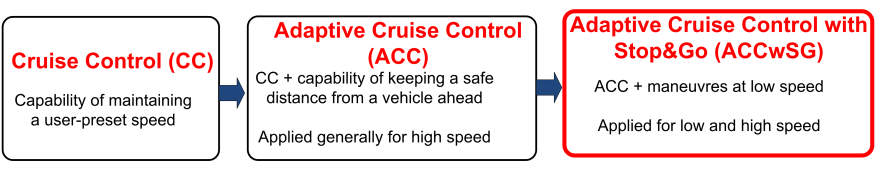

Fig. 3. ACCwSG concept

ACCwSG scheme proposed in this paper is presented in the figure 4 . The aim is to keep the $d_{\text {ref }}$, which is an inter-vehicular distance to keep when vehicles move. At the full stop of both vehicles, the minimal safety distance $d_{\text {min safety }}$ must be respected. In order to carry out the ACCwSG, the following control scheme is proposed (cf. figure 5). In the control scheme the following notations were used:

- $v_{L V}, v_{F V}$ : leader and follower vehicle speeds,

- $v_{\text {opt }}$ : sub-optimal speed profile obtained by DP-based algorithm solutions,

- $T_{E M \text { setpoint }}, T_{H M \text { setpoint }}$ : sub-optimal torque of EM and $\mathrm{HM}$, respectively, related to $v_{o p t}$,
- $d$ current inter-vehicular distance,

- Distance to keep block: provides inter-vehicular distance $d_{r e f}$ to maintain between the vehicles,

- Safe speed block: calculates the speed set point $v_{r e f}$ needed to keep the reference distance $d_{\text {ref }}$.

OPD-DP (Optimal Profiles Database based on DP) block contains the optimal energy management strategy and optimal speed profiles MDD, which is the key element of the paper. This work focuses on the development of the optimal speed profiles and energy management strategy for ACCwSG.

In this paper it is assumed that distance $d$ between the vehicles is known. In order to avoid collisions with the preceding car, the bus must follow the reference speed $v_{r e f}$ which must consider the safety requirements. The reference distance $d_{r e f}$ to keep between the vehicles consists of:

$$
d_{\text {ref }}=d_{\text {min safety }}+d_{v}
$$

The distance $d_{\text {min safety }}$ is minimal distance to maintain at full stop of both vehicles. The distance $d_{v}$ is defined by the constant time headway policy [18]:

$$
d_{v}=T_{h} v
$$

where $T_{h}$ is a constant headway time which approximates driver's reaction time (generally, between 0.8 and $2.5 \mathrm{~s}$ ), and $v$ is current speed. In case when $d>d_{\text {ref }}$, the bus functions in Cruise Control (CC) mode and $v_{r e f}=v_{C C}$, where $v_{C C}$ a driver preset cruise speed. In case when $d \leq d_{\text {ref }}$, reference speed $v_{r e f}$ is calculated as follows:

$$
v_{\text {ref }}=\sqrt{v^{2}+2 a_{\text {relative }} S}
$$

where $a_{\text {relative }}$ is a relative acceleration/deceleration between two vehicles:

$$
a_{\text {relative }}=\frac{d v_{L V}}{d t}-\frac{d v_{F V}}{d t}
$$

And distance $S$ is calculated as follows:

- Case 1: $d \geq d_{\text {min safety }}$

$$
S=\left(d_{\min \text { safety }}+d_{v}\right)-d
$$

- Case 2: $d<d_{\text {min safety }}$

$$
S=d_{\text {min } \text { safety }}-d
$$

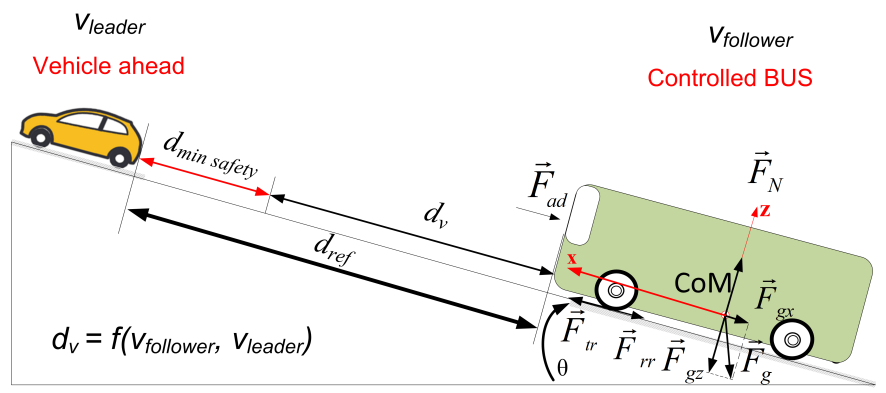

Fig. 4. Forces acting on the bus and ACCwSG scheme 
To reach the reference speed $v_{r e f}$, HEV has multiple choices. The problem of choosing an optimal speed profile and appropriate mode to minimize the fuel consumption during ACCwSG phases is a challenging issue. For that purpose, sub-optimal ACCwSG algorithm is developed using DP. Below the optimization criteria is formulated and the offline and online optimization strategies are proposed.

\section{PROPOSED SUB-OPTIMAL ACCWSG BASED ONLINE DP ALGORITHMS}

This section is dedicated to the development of a simultaneous speed profile optimization and an energy management strategy in offline and online mode. The formulation of the multi-criteria optimization is given in IV-A. The procedure of the decision tree construction is described in details in section IV-B. The developed offline algorithm permits to move to the online implementation by means of the Optimal Profiles Database (cf. section IV-C), which generates the speed profile and its energy management strategy, depending on the road profile, current bus state and velocity set-point.

\section{A. Overall Multi-Criteria Optimization formulation}

The objective of the optimal control problem is to find the optimal bus velocity profile and energy split between the actuators for a given trajectory. The optimization is performed in the spatial domain by means of the following transformation (conversion from time domain into spatial domain) [10]:

$$
a=\frac{\mathrm{d} v}{\mathrm{~d} t}=\frac{\mathrm{d} v}{\mathrm{~d} D} \frac{\mathrm{d} D}{\mathrm{~d} t}=\frac{\mathrm{d} v}{\mathrm{~d} D} v
$$

In that case the dynamics of the BUSINOVA given in equation (4) could be re-written with the following equation:

$$
\frac{d v}{d D}=\frac{1}{\left(M+M_{e q}\right) v} H
$$

As any problem of optimization, it is important to define optimization criteria. In our case, the criteria is defined in order to minimize the fuel mass consumed $\dot{m}_{f u e l}[19]$ and the electric power $P_{E M}$ consumed by the electric motor during the trip $D$ :

$$
J_{D}=\alpha J_{1}+(1-\alpha) J_{2}
$$

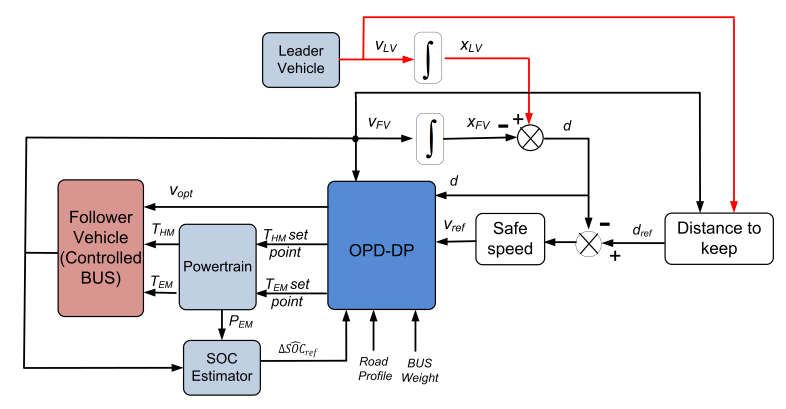

Fig. 5. Proposed control architecture and its efficient energy management strategy for ACCwSG maneuvers where:

$$
J_{1}=\int_{0}^{D_{f}} \frac{Q_{L H V} \dot{m}_{f u e l}\left(P_{I C E}(D), P_{H M}(D), D_{H M}\right)}{v(D)} d D
$$

with $Q_{L H V}$ is lower heating value of a used fuel, $\dot{m}_{f u e l}$ fuel mass consumed is a function of ICE power $P_{I C E}$, as well as of HM power $P_{H M}$ and HM displacement $D_{H M}$ [19];

$$
J_{2}=\int_{0}^{D_{f}} \frac{P_{E M}(D)}{v(D)} d D
$$

with $P_{E M}(D)$ EM power. $D_{f}$ is the total traveled distance; $v$ is the bus speed; $\alpha$ is a constant weight coefficient such as $\alpha \in\left[\begin{array}{ll}0 & 1\end{array}\right]$.

The minimization of the cost function (14) is the subject to the bus dynamical model (13), as well as to the constraints imposed on the control input and state during the performed optimization.

The electric battery should keep the charge over its minimal permissible charge level during the whole operational cycle. That is why the constraint on the battery usage during the trip is imposed by the introduction of Predictive $S O C$ (P-SOC). In this work, the principle idea is to consider that a better usage of the electric energy is such that it is available until the end of the day (during 8 hours operational cycle), and this is considered as an optimal functioning of the bus. The working hypothesis behind this assumption is to use the maximum amount of energy that can be consumed from the battery in one day driving so that the battery energy is spread as uniformly as possible in one working day. This implies the smooth battery discharging rate (C-rate), avoidance of the high or low $S O C$ and excessive depth of charge, which lead to a high rate of battery capacity loss [20]. As Li-ion batteries represent a big part of a vehicle cost, the clear interest is to prolongate the battery life. For that purpose a $S O C$ estimator based on Kalman Filter [21] is proposed.

\section{B. Offline Optimization Strategy based on DP}

DP formulation: The optimization method used to solve the given optimal control problem is based on DP [22] [23], which provides the global optimal solution over a given trip. The algorithm proceeds from 0 to $K$ steps in order to minimize the following cost function (Hamilton-JacobiBellman (HJB) equation):

$$
J_{k}\left(v_{k}\right)=\min _{u_{k} \in \mathbb{U}_{D}}\left\{g_{k}\left(v_{k}, u_{k}, \Delta D_{k}\right)+J_{k-1}\left(f\left(v_{k}, u_{k}\right)\right)\right\}
$$

where

- $J_{k}\left(v_{k}\right)$ is the cost-to-go function from step 0 to step $K$ starting from $v_{0}$ with initial cost $J_{0}\left(v_{0}\right)=g_{0}\left(v_{0}\right)=0$.

- $g_{k}\left(v_{k}, u_{k}, \Delta D_{k}\right)$ is the cost-to-go from state $i$ to state $j$.

- $J_{k-1}\left(f\left(v_{k}, u_{k}\right)\right)$ is the total cost starting from the initial state to the state $i$.

- $u_{k} \in \mathbb{U}_{D}$ is the control input determining the velocity to go from the state $i$ to the next state $j$. 
The given optimization is aimed to solve two main problems simultaneously: (i) find the optimal speed profile from $D_{0}$ to $D_{f}$ in time $t_{f}$, minimizing the electric energy and fuel consumption; (ii) find the optimal power split strategy to the speed profile in order to provide the optimal functioning mode (cf. section II) and the percentage of the contribution of each motor (EM and HM) in order to move the hybrid bus.

The paragraphs below detail the resolution of the nonlinear optimization control problem formulated in the spatial domain by using DP algorithm. A set of points defines the route. Namely, the route consists of the points $P=$ $\left[p_{0}, \quad p_{1}, \quad p_{2}, \quad \ldots, \quad p_{K}\right]$. Every point $p_{k} \in P$ for $k=$ $0,1,2, \ldots, K$ has its own characteristics: $p_{k}=\left[x_{k}, y_{k}, \theta_{k}\right]$, where $x_{k}$ longitudinal position, $y_{k}$ lateral position, $\theta_{k}$ road slope angle. The given route of the length $D$ is divided into $K$ segments of the sample length $\Delta D$. Depending on the acceleration/deceleration limits and on the $\Delta D$ segment length, the velocity $v\left(D_{k}\right)$ for a given segment $D_{k}$ can increase or decrease with a fixed step $\Delta v$. The maximum number of $\Delta v$ is equal to $2 N_{v}+1$ :

$$
\mathbb{V}=\left\{-N_{v} \Delta v, \ldots, \quad 0, \quad \Delta v, \quad \ldots, \quad N_{v} \Delta v\right\}
$$

where $N_{v} \in \mathbb{N}$.

DP-SEO algorithm flowchart: Figure 6 shows the proposed DP based Speed and Energy Optimization (DP-SEO) algorithm. The proposed DP-SEO has the following main steps.

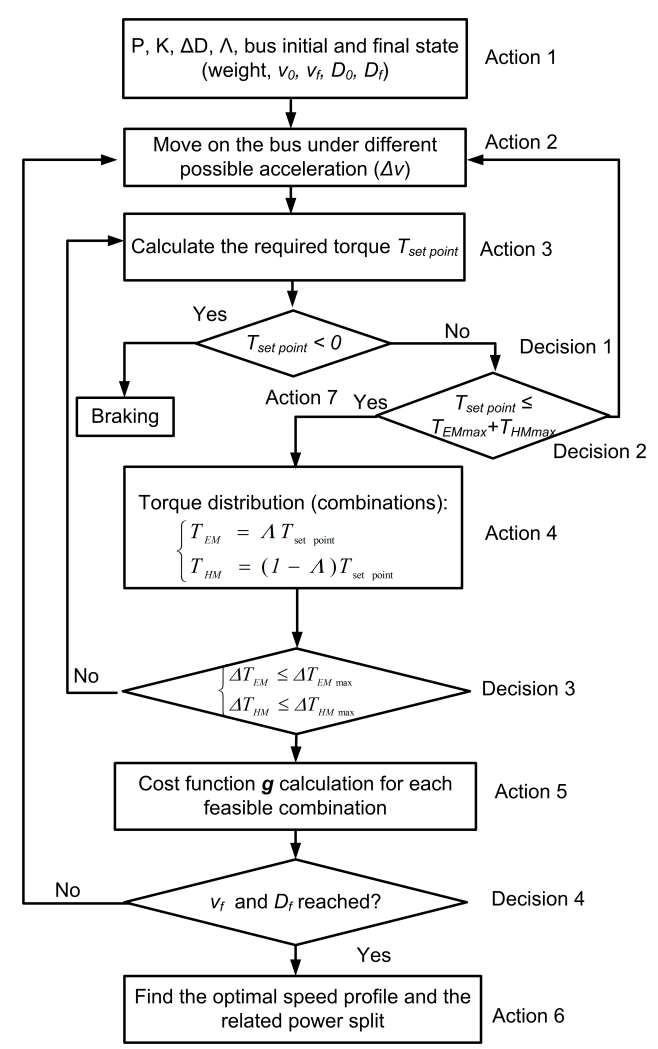

Fig. 6. Block diagram of the Dynamic Programming based Speed and Energy Optimization (DP-SEO)
Action 1. It initializes the road profile $P$, distance discretization step $\Delta D, K$ number of $\Delta D$, a set of maximum permissible values of speed increment $\Delta v$. To perform a simultaneous bus speed profile and its power split optimization, the specific vector $\Lambda=\{0,0.1,0.2, \ldots, 1\}$ is assigned with $\operatorname{Card}(\Lambda)=m$ cardinal number of the set $\Lambda$, which corresponds to the contribution of the EM and HM in traction. Each state $\nu_{j} \equiv\left[P_{k}, v_{j}, t_{j}, \operatorname{Parent}\left(v_{j}\right)\right]$, with $v_{j}$ speed, $t_{j}$ time, Parent $\left(v_{j}\right)$ previous state.

Action 2. It attributes an acceleration $\Delta v$.

Action 3. It calculates the required torque $T_{\text {setpoint }}$.

Decision 1. If $T_{\text {setpoint }}$ is negative, the braking is applied (cf. Action 7).

Decision 2. If $T_{\text {setpoint }}$ is less than the maximum torque that can be produced by EM and HM, then go Action 4.

Action 4. It calculates the possible torque ratio combinations according to $\Lambda$.

Decision 3. The EM and the engine, which drives the HM, have different dynamic characteristics. EM is capable of delivering a high starting torque. Unlike EM, engine cannot provide torque at zero speed and it produces maximum power at a certain speed [24]. The efficiency of the engine is very much dependent on the operating point in the engine's performance map. Thus, to go from one state to another not all the $\Delta T$ are feasible. To take this constraint into account, the maximum values $\Delta T_{E M_{\max }}$ and $\Delta T_{H M_{\max }}$ are introduced. If the demanded torque is higher that these values, then the development of the decision tree in that direction is stopped. Otherwise, go to Action 5.

Action 5. For the feasible combinations the cost-to-go function $g_{k}\left(v_{k}, u_{k}, \Delta D_{k}\right)$ is calculated (cf. equation (17)).

Decision 4. If the final conditions are reached, then go to Action 6. Otherwise, go to Action 2.

Action 6. The minimum cost function is calculated according to equation (17). Optimal speed profile and related power split are calculated. In this work, DP is used to obtain the minimal cost leading the hybrid bus from its initial state to the final one.

Action 7. A part of the kinetic energy is regenerated to charge the battery, and the rest is dissipated via mechanical brake. If the vehicle speed is high enough then the kinetic energy can be regenerated (limited to the maximum torque $T_{\text {regenmax }}$ produced by EM and depending on the current vehicle speed). No regeneration is possible under $v<10$ $\mathrm{km} / \mathrm{h}$. In future work, the regenerative aspect and dynamic will be studied in more details.

DP-SEO results analysis: In order to validate the proposed offline optimization, it is performed several DP-SEO in different elementary conditions of slope and desired velocity progress (constant speed $\left(v_{0}=v_{f}\right)$, acceleration $\left(v_{0}<v_{f}\right)$ and deceleration $\left(v_{0}>v_{f}\right)$ phases $)$ for a fixed distance $D$, $K$ number of $\Delta D$ and $\Lambda$. Figures 7-8 show the average energy consumption for different slopes $\theta$ for aforementioned phases. It can be seen that the energy consumption increases with a bigger $\theta$. A bigger bus weight also increases the fuel consumption. To keep the cruise speed on the downhill slope (negative $\theta$ ), the acceleration produced by the bus' 
own dynamics is enough (sometimes even excessive and it is necessary to apply a negative torque). So on the downhill slope the EM part is $100 \%$ for deceleration and cruise speed phases. When accelerating, the EM torque goes to its peak with the increase of the uphill slope, and it is complemented by the HM (via ICE), so we can see that EM ratio increases.

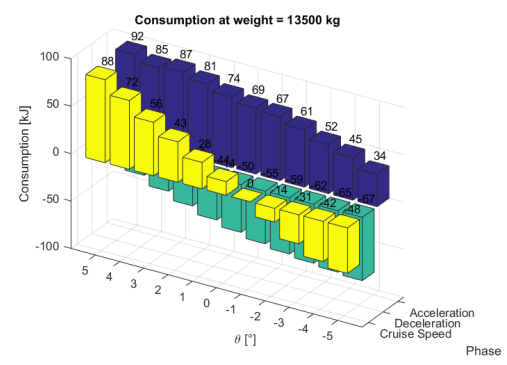

Fig. 7. Average energy consumption for different slopes

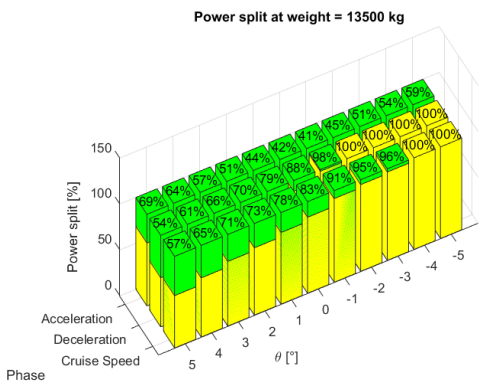

Fig. 8. Average power split for different slopes

\section{Online Sub-Optimal Strategy for ACCwSG}

Even if the proposed strategy of the DP-SEO gives optimal results (according to the chosen discretization parameter $\Delta D, \Delta v$ and $\Lambda$ (cf. section IV-B), all the possible states are explored and the global minimum is found), nevertheless it is highly time consuming.

However, from the results of the optimization presented above, the optimal offline solutions can be used online in order to generate the sub-optimal solutions. For that purpose, it is proposed to follow the following protocol, which consists to generate the optimal profiles offline for a chosen prediction horizon $\Delta d$ with different $\Delta v$ for several constant masses and slopes. Different scenarios (for acceleration, deceleration, maintaining the same speed, provided that the final $D_{f}$ is reached) were simulated and for each simulation configuration, an optimal speed profile and related power split are obtained.

The Optimal Profiles Database based on DP (OPD-DP) was built for all the possible combination of velocities from 0 to $15 \mathrm{~m} / \mathrm{s}$ with $\Delta v=1 \mathrm{~m} / \mathrm{s}$, the bus weight varies from 13 to 15 tonnes with $\Delta M=0.2$ tonne, and the slope angle value ranges from $-5^{\circ}$ to $5^{\circ}$ with $\Delta \theta=1^{\circ}$. A bigger discretization step of the parameters leads to a higher energy consumption variation for a different set of parameters (weight, slope, speed), thus leading to bigger uncertainty of the powersplit in the configurations which are not belonging to the database. Smaller discretization step ensures smoother transition between different sets of parameters. However, too small discretization step may lead to very heavy database. The database discretization step presented in the paper is a compromise between these two extremes. Based on the offline optimal solutions, OPD-DP will be used to generate the speed profiles and the related power split in a real-time.

The multi-dimensional OPD-DP is used for online speed profile and its power split generation. The scheme of the online control implementation is presented in figure 5. The inputs of the multi-dimensional OPD-DP are the road profile $(x, y, \theta)$, the bus weight, the current speed and the driver's reference (desired) speed, and the current vehicle's position. This block generates the sub-optimal speed profile reference and torque split depending on the current state of the vehicle and the road profile. The bus weight is considered constant for a single trip between two stops, thus the weight is susceptible to change only after each stop. The reference speed $v_{\text {ref }}$ must be followed as precise as possible due to the safety measures, ensuring the collision avoidance. The OPD-DP generates the sub-optimal profile references $v_{o p t}$, as well as $T_{E M}$ and $T_{H M}$ set points. The set point torques sent to EM and HM local controllers, which generate the control input to the motors $\left(I_{E M}\right.$ EM current, $D_{H M} \mathrm{HM}$ displacement).

Knowing that the multi-dimensional OPD-DP has only discrete values of each parameters (slope $\theta$, weight $M$, etc.), it is very important to have a mean to use this MDD even for values not belonging to it. For this purpose, several techniques are proposed in order to extend the knowledge existing in the MDD.

\section{Multi-Dimensional Interpolation Method}

1) MinMax Value: the values which are closer to the upper or the lower bound values are extracted for the mass, slope, speed profile and energy management split.

2) Average Value: the values which are closer to the current mass and slope are extracted. The average value of the speed between the upper and the lower bounds are calculated. The average value of the power split is applied as well.

3) Linear Multi-Dimensional (LMD) interpolation method [25]. As a desired velocity value $v$ lies between two values in the OPD-DP, the weighted sum of the lower bound speed $v_{U B}$ and the upper bound speed $v_{L B}$ are applied to generate the speed profile:

$$
v_{o p t}=\zeta_{v} v_{L B}+\left(1-\zeta_{v}\right) v_{U B}
$$

The weight coefficient $\zeta_{v}$ is obtained as follows:

$$
\zeta_{v}=1-\frac{v-v_{L B}}{v_{U B}-v_{L B}}
$$


The weight coefficients applied to calculate the corresponding power split $\Lambda_{\text {opt }}$, depend on the current speed $v$, the bus weight $M$, and the road slope $\theta$. This results into three dimensional interpolation function given as follows:

$$
\begin{gathered}
\Lambda_{o p t}(v, M, \theta)=\zeta_{v} \zeta_{M} \zeta_{\theta} \Lambda\left(v_{L B}, M_{L B}, \theta_{L B}\right)+ \\
\left(1-\zeta_{v}\right) \zeta_{M} \zeta_{\theta} \Lambda\left(v_{U B}, M_{L B}, \theta_{L B}\right)+ \\
\zeta_{v}\left(1-\zeta_{M}\right) \zeta_{\theta} \Lambda\left(v_{L B}, M_{U B}, \theta_{L B}\right)+ \\
\zeta_{v} \zeta_{M}\left(1-\zeta_{\theta}\right) \Lambda\left(v_{L B}, M_{L B}, \theta_{U B}\right)+ \\
\left(1-\zeta_{v}\right)\left(1-\zeta_{M}\right) \zeta_{\theta} \Lambda\left(v_{U B}, M_{U B}, \theta_{L B}\right)+ \\
\left(1-\zeta_{v}\right) \zeta_{M}\left(1-\zeta_{\theta}\right) \Lambda\left(v_{U B}, M_{L B}, \theta_{U B}\right)+ \\
\zeta_{v}\left(1-\zeta_{M}\right)\left(1-\zeta_{\theta}\right) \Lambda\left(v_{L B}, M_{U B}, \theta_{U B}\right)+ \\
\left(1-\zeta_{v}\right)\left(1-\zeta_{M}\right)\left(1-\zeta_{\theta}\right) \Lambda\left(v_{U B}, M_{U B}, \theta_{U B}\right)
\end{gathered}
$$

where $\Lambda_{o p t}$ is the power split vector, and weight coefficients $\zeta_{M}$ and $\zeta_{\theta}$ are calculated as follows:

$$
\begin{gathered}
\zeta_{M}=1-\frac{M-M_{L B}}{M_{U B}-M_{L B}} \\
\zeta_{\theta}=1-\frac{\theta-\theta_{L B}}{\theta_{U B}-\theta_{L B}}
\end{gathered}
$$

with indexes $L B$ - lower bound and $U B$ - upper bound of the corresponding parameter.

To illustrate how this method works in $3 \mathrm{D}$, let us fix speed parameters, and suppose that the powersplit $\lambda$ change depends only on the variation of the bus weight and the road slope. Figure 9 illustrates the case when for different combinations of weight and slope angle, we have four known values of $\lambda$ stored in the OPD-DP. Now let us address the case when weight and slope angle values lie in between the known data. This method permits to calculate the corresponding powersplit.

The comparison of these three methods showed that better results, in terms of reaching the desired speed and the energy minimization, were demonstrated by means of LMD method. The next section presents the results using this method.

\section{SIMULATION RESULTS}

For the simulation scenario it is considered that the Bus follows a car. In the first stage, the car performs three Stop\&Go situations. Afterwards, the preceding car accelerates, so that the distance between the vehicles becomes

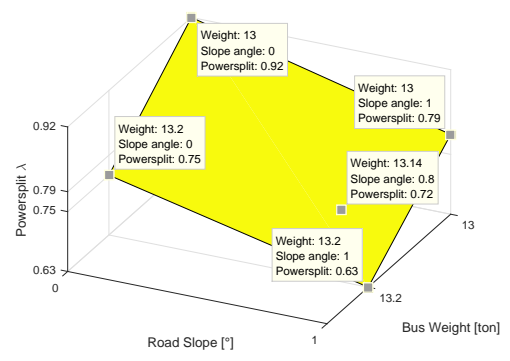

Fig. 9. Linear Multi-dimensional Interpolation method illustration

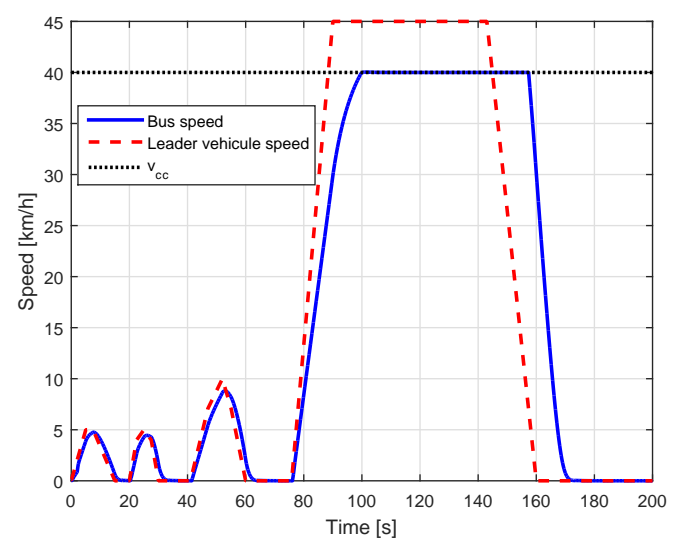

Fig. 10. Speed of the Leader Vehicle and the Bus

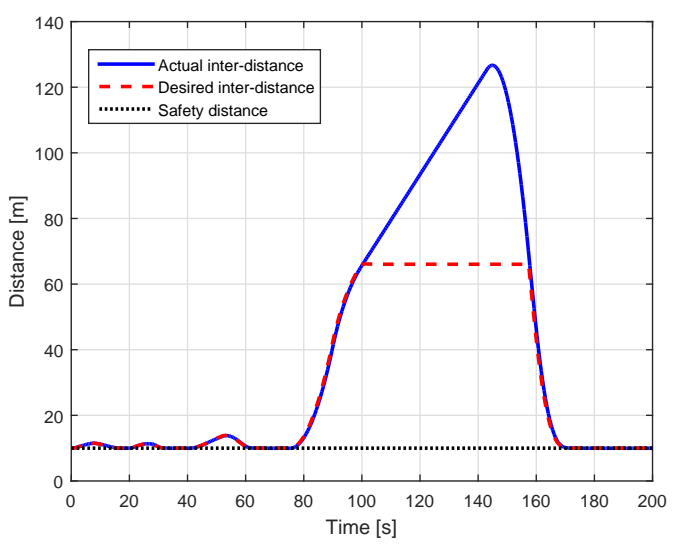

Fig. 11. Inter-vehicular distance

$d>d_{\text {ref }}$ and the bus switches to Cruise Control phase and moves with its preset speed.

Figures 10 and 11 demonstrate the obtained results. We can see that the speed profile of the Bus is smooth (cf. figure 10). The minimal safety distance $d_{\min \text { safety }}=10 \mathrm{~m}$ (cf. figure 4). Figure 11 shows that $d_{\text {min safety }}$ is always respected. Once the inter-vehicular distance $d>d_{\text {ref }}$ the bus keeps its cruise speed of $40 \mathrm{~km} / \mathrm{h}$. When the preceding car starts to decelerate, the bus does not change its speed until $d=d_{\text {ref }}$. Then it decelerates until its full stop. The energy consumption is compared in the Table I. For comparison, the online sub-optimal strategy was compared to two Energy Management Strategies (EMS): StateFlow and offline DP. StateFlow [26] is a switching strategy between operational modes, according to the determined transition conditions. Offline DP shows the best performance, but spends more than 1082 seconds to obtain the result and it implies to have the knowledge of the overall vehicle path in advance. Unlike offline DP, online sub-optimal strategy can be used in realtime applications. The obtained solution, in terms of energy consumption, corresponds to the best $10 \%$ of all the possible solutions found by offline DP. Several other simulations with different behavior of the Leader vehicle have been performed 
TABLE I

COMPARISON OF DIFFERENT EMS

\begin{tabular}{|l|l|l|l|}
\hline EMS & Fuel [1] & $\Delta S O C[\%]$ & Energy $[\mathrm{kJ}]$ \\
\hline StateFlow & 0.022 & 0.39 & 3917 \\
\hline Online OPD-DP & 0.018 & 0.36 & 3674 \\
\hline Offline DP & 0.017 & 0.34 & 2581 \\
\hline
\end{tabular}

and confirm that the proposed online sub-optimal strategy always produces smooth and in average always among the best $10 \%$ of the obtained solutions using offline DP.

\section{CONCLUSIONS AND PROSPECTS}

This paper presented an overall control architecture and its dedicated energy management strategy for HEV for low consumption ACCwSG maneuvers. The developed overall architecture finds a highly practical application in ACCwSG for the studied heavy urban hybrid bus, which is always subject to frequent starts and stops phases (maneuvers which are generally subject to important energy consumption). An appropriate offline DP-SEO algorithm permitting to simultaneously generate an optimal speed profile and its power split strategy is proposed in this paper in order to ensure: the electric energy and fuel economy, while respecting passengers comfort (mainly by limiting the maximal acceleration/deceleration range and the jerking aspects) and taking into account the electric motor and engine dynamics. Afterward, the optimal offline DP-SEO solutions, for different elementary scenarios, have been collected into a multidimensional OPD-DP for online implementation in a priori unknown road and for any traffic conditions, as in the reallife case this information is not available beforehand but can be detected by the on-board sensors. The OPD-DP has been better operated while using mainly a specific LMD algorithm (as reliable interpolation function) in order to deal with more exhaustive HEV situations/states. The obtained results using the proposed algorithms for ACCwSG show relevant results since it provides smooth bus speed profiles and relevant energy management, minimizing the fuel consumption.

It is planned in near future to use stochastic decision process (as MDP) and predictive mechanisms in order to improve the proposed online strategy based on OPD-DP.The overall architecture is also planned to be implemented soon in the real bus.

\section{REFERENCES}

[1] Y. L. Murphey, "Intelligent vehicle power management: An overview," in Computational Intelligence in Automotive Applications, pp. 169190, Springer, 2008.

[2] J. Jonsson, "Fuel optimized predictive following in low speed conditions," 2003

[3] K. Yu, J. Yang, and D. Yamaguchi, "Model predictive control for hybrid vehicle ecological driving using traffic signal and road slope information," Control theory and technology, vol. 13, no. 1, pp. 17-28, 2015.

[4] K. Yi, J. Hong, and Y. Kwon, "A vehicle control algorithm for stopand-go cruise control," Proceedings of the Institution of Mechanical Engineers, Part D: Journal of Automobile Engineering, vol. 215, no. 10, pp. 1099-1115, 2001.

[5] S. Kim, "Design of the adaptive cruise control systems: An optimal control approach," 2012.
[6] G. Rousseau, Véhicule hybride et commande optimale. $\mathrm{PhD}$ thesis, École Nationale Supérieure des Mines de Paris, 2008.

[7] B.-C. Chen, Y.-Y. Wu, and H.-C. Tsai, "Design and analysis of power management strategy for range extended electric vehicle using dynamic programming," Applied Energy, vol. 113, pp. 1764-1774, 2014.

[8] S. Kitayama, M. Saikyo, Y. Nishio, and K. Tsutsumi, "Torque control strategy and optimization for fuel consumption and emission reduction in parallel hybrid electric vehicles," Structural and Multidisciplinary Optimization, vol. 52, no. 3, pp. 595-611, 2015.

[9] N. Ouddah, L. Adouane, R. Abdrakhmanov, and E. Kamal, "Optimal energy management strategy of plug-in hybrid electric bus in urban conditions," in ICINCO17, International Conference on Informatics in Control, Automation and Robotics, Madrid-Spain, 26-28 July, 2017.

[10] E. Ozatay, S. Onori, J. Wollaeger, U. Ozguner, G. Rizzoni, D. Filev, J. Michelini, and S. Di Cairano, "Cloud-based velocity profile optimization for everyday driving: A dynamic-programming-based solution," Intelligent Transportation Systems, IEEE Transactions on, vol. 15 , no. 6 , pp. 2491-2505, 2014.

[11] P. Tokekar, N. Karnad, and V. Isler, "Energy-optimal trajectory planning for car-like robots," Autonomous Robots, vol. 37, no. 3, pp. 279 300, 2014.

[12] W. Dib, A. Chasse, P. Moulin, A. Sciarretta, and G. Corde, "Optimal energy management for an electric vehicle in eco-driving applications," Control Engineering Practice, vol. 29, pp. 299-307, 2014.

[13] D. Pei and M. J. Leamy, "Dynamic programming-informed equivalent cost minimization control strategies for hybrid-electric vehicles," Journal of Dynamic Systems, Measurement, and Control, vol. 135, no. 5, p. 051013,2013

[14] Z. Song, H. Hofmann, J. Li, X. Han, and M. Ouyang, "Optimization for a hybrid energy storage system in electric vehicles using dynamic programing approach," Applied Energy, vol. 139, pp. 151-162, 2015.

[15] R. Abdrakhmanov and L. Adouane, "Dynamic programming resolution and database knowledge for online predictive energy management of hybrid vehicles," in ICINCO17, International Conference on Informatics in Control, Automation and Robotics, Madrid-Spain, 26-28 July, 2017.

[16] K. Ç. Bayindir, M. A. Gözüküçük, and A. Teke, "A comprehensive overview of hybrid electric vehicle: Powertrain configurations, powertrain control techniques and electronic control units," Energy Conversion and Management, vol. 52, no. 2, pp. 1305-1313, 2011.

[17] Y. Cheng, P. Lataire, et al., "Research and test platform for hybrid electric vehicle with the super capacitor based energy storage," in Power Electronics and Applications, 2007 European Conference on, pp. 1-10, IEEE, 2007.

[18] P. Shakouri, J. Czeczot, and A. Ordys, "Simulation validation of three nonlinear model-based controllers in the adaptive cruise control system," Journal of Intelligent \& Robotic Systems, vol. 80, no. 2, pp. 207-229, 2015.

[19] X. Zeng, "Improving the energy density of hydraulic hybridvehicle (hhvs) and evaluating plug-in hhvs," 2009.

[20] L. Tang, G. Rizzoni, and S. Onori, "Energy management strategy for hevs including battery life optimization," IEEE Transactions on Transportation Electrification, vol. 1, no. 3, pp. 211-222, 2015.

[21] G. Welch and G. Bishop, "An introduction to the kalman filter," 1995

[22] R. E. Bellman and S. E. Dreyfus, Applied dynamic programming. Princeton university press, 2015.

[23] D. P. Bertsekas, Dynamic programming and optimal control, vol. 1 Athena Scientific Belmont, MA, 1995.

[24] P. Pisu and G. Rizzoni, "A comparative study of supervisory control strategies for hybrid electric vehicles," IEEE Transactions on Control Systems Technology, vol. 15, no. 3, pp. 506-518, 2007.

[25] S. M. LaValle, Planning algorithms. Cambridge university press, 2006.

[26] A. Hajizadeh and M. A. Golkar, "Intelligent power management strategy of hybrid distributed generation system," International Journal of Electrical Power \& Energy Systems, vol. 29, no. 10, pp. 783-795, 2007 